Präv Gesundheitsf 2015 • 10:199-205

DOI 10.1007/s11553-015-0496-z

Online publiziert: 6. Mai 2015

(c) Die Autor(en) 2015. Dieser Artikel ist auf

Springerlink.com mit Open Access verfügbar
Bianca Biallas · Philip Ashton · Christiane Wilke - Tobias Elis · Ingo Froböse

Institut für Bewegungstherapie und bewegungsorientierte Prävention und Rehabilitation,

Deutsche Sporthochschule Köln, Köln, Deutschland

\title{
Tätigkeits- und altersbezogene Analyse von Arbeitsfähigkeit und körperlicher Aktivität
}

lichen Erfahrung dazu bei, die Zukunft des Unternehmens zu sichern. Der demografische Wandel muss in diesem $\mathrm{Zu}-$ sammenhang von Politik, Wirtschaft und Forschung als Zukunftsaufgabe für die gesamte Gesellschaft begriffen werden [23].

\section{Arbeitsfähigkeit}

Die muskuloskelettalen Erkrankungen nehmen Einfluss auf das Wohlbefinden und die Arbeitsfähigkeit des Arbeitnehmers und erhöhen somit das Risiko von krankheitsbedingten Ausfallzeiten und Frühverrentung [18]. Studien zeigen, dass die Art der beruflichen Tätigkeit Einfluss auf die Arbeitsfähigkeit hat, was u. a. im unterschiedlichen Ausmaß der körperlichen Aktivität und körperlichen Beanspruchung bei der Arbeit begründet liegt [19]. Arbeitnehmer mit mentaler beruflicher Tätigkeit weisen eine höhere Arbeitsfähigkeit auf, als Arbeitnehmer aus körperlichen Berufen [8]. Zudem steigt die Wahrscheinlichkeit einer schlechten Arbeitsfähigkeit mit höherem Alter, obgleich diese auch im höheren Alter durch Maßnahmen (u. a. Förderung der Gesundheit, Steigerung der beruflichen Handlungskompetenz, Verbesserung der Arbeitsorganisation) wieder gesteigert werden [16].

Faktoren, die die Arbeitsfähigkeit beeinflussen, werden verstärkt ab dem mittleren Erwerbsalter deutlich [16], darunter auch das Ausmaß an körperlicher Aktivität. Es gilt daher, nicht nur die Arbeitsfähigkeit aller Arbeitnehmer gleichermaßen zu fördern bzw. zu erhalten, sondern auch einen besonderen Fokus bereits auf das frühe Erwerbsalter zu richten, um individuelle Faktoren und Jobbedingungen in einen Konsens zu bringen.

\section{Körperliche Aktivität}

Die fortschreitende Technologisierung in der Gesellschaft hat zu einer Senkung der körperlichen Beanspruchungen im Alltag und im Beruf geführt. Die Folgen dieses technologischen Fortschritts äußern sich in einer erhöhten körperlichen Inaktivität und Prävalenz verschiedener Zivilisationskrankheiten (Adipositas, Diabetes mellitus [20]). Die körperliche Aktivität nimmt i. Allg. altersprogressiv und geschlechtsunabhängig ab: Der Großteil der deutschen Männer und Frauen erreicht die vom „American College of Sports Medicine" (ACSM) vorgegebene Mindestmenge an körperlicher Aktivität von mindestens für $2,5 \mathrm{~h}$ pro Woche mäßiger körperlicher Aktivität [15]. In der Altersgruppe der 18- bis 29-Jährigen sind 62,2\% der Frauen und 40,7\% der Männer weniger als $2,5 \mathrm{~h}$ pro Woche aktiv, in den Altersgruppen 30 bis 44 Jahre und 45 bis 64 Jahre sind es $60,6 \%$ und $54,2 \%$ bzw. $59,9 \%$ und $59,9 \%$ respektive [26]. Dabei sind Personen ab 50 Jahre nicht notwendigerweise weniger körperlich aktiv, aber es besteht mit steigendem Alter eine höhere Wahrscheinlichkeit dafür, dass die Aktivitätsempfehlungen nicht erreicht werden, insbesondere bei Frauen [21].

Grundsätzlich ist die körperliche Aktivität von der körperlichen Arbeit abzugrenzen. Während körperliche Beanspruchungen bei der Arbeit mitunter einseitig und hochintensiv sein können, werden der körperlichen Aktivität, insbesondere die moderat-intensive Aktivität, gesundheitsförderliche Attribute zugeschrieben. So weisen Beschäftigte aus körperlichen Arbeitsbereichen zwar höhere Beanspruchungen auf, als vornehmlich geistig Tä- 


\begin{tabular}{|c|l|l|}
\hline Punkte & Arbeitsfähigkeit & Ziel von Maßnahmen \\
\hline $7-27$ & Schlecht & Arbeitsfähigkeit wiederherstellen \\
$28-36$ & Mittelmäßig & Arbeitsfähigkeit verbessern \\
$37-43$ & Gut & Arbeitsfähigkeit unterstützen \\
$44-49$ & Sehr gut & Arbeitsfähigkeit erhalten \\
\hline
\end{tabular}

Abb. $1<$ Einstufung und Bewertung der Arbeitsfähigkeit [8]

\begin{tabular}{|c|c|}
\hline Aktivitätsniveau & Kriterien \\
\hline hoch & $\begin{array}{l}\text { Die Person erfüllt die folgenden Kriterien: } \\
\text { - Intensive körperliche Aktivitäten an mindestens } 3 \text { Tagen mit insgesamt } \\
\geq 1500 \text { MET-Minuten pro Woche oder } \\
\text { - } 7 \text { Tage in jeglicher Kombination von Geh-, moderaten - oder intensiven } \\
\text { Aktivitäten mit einem Minimum von } 3.000 \text { MET-Minuten pro Woche }\end{array}$ \\
\hline moderat & $\begin{array}{l}\text { Die Person ist nicht hoch aktiv, erfüllt jedoch die folgenden Kriterien: } \\
\text { - } 3 \text { oder mehr Tage für mindestens für } 20 \text { Minuten intensiv körperlich } \\
\text { aktiv (mind. } 480 \text { MET-Minuten) oder } \\
\text { - } 5 \text { oder mehr Tage in einer moderaten intensität für mindestens } 30 \\
\text { Minuten gegangen (mind. } 600 \text { MET-Minuten) oder } \\
\text { - } 5 \text { oder mehr Tage in jeglicher Kombination von Geh-, moderaten oder } \\
\text { intensiven Aktivitäten mit einem Minimum von } 600 \text { MET-Minuten pro } \\
\text { Woche }\end{array}$ \\
\hline niedrig & $\begin{array}{l}\text { Die Person erfüllt keine der oben genannten Kriterien und fällt in diese } \\
\text { kategorie }\end{array}$ \\
\hline
\end{tabular}

Abb. $2 \Delta$ Aktivitätsniveau anhand der erreichten MET-Minuten pro Woche [2]

tige. Körperlich beanspruchende Berufe bedeuten demnach nicht zwangsläufig eine höhere gesundheitsförderliche Wirkung.

\section{Ziel und Fragestellung}

Das Ziel dieser Untersuchung ist die Analyse der Arbeitsfähigkeit und körperlichen Aktivität bei Arbeitnehmern eines mittelständischen Unternehmens. Durch den besonders hohen Anteil der mittelständischen Unternehmen in der Wirtschaft kann ein repräsentatives, übertragbares Ergebnis erzielt werden. Es wird untersucht, ob sich Arbeitnehmer in diesen Faktoren hinsichtlich Alter und Tätigkeitsbereich (kaufmännisch/gewerblich) unterscheiden. Daraus leiten sich folgende Fragestellungen ab:

- Unterscheiden sich Arbeitnehmer im frühen, mittleren und späten Er- werbsalter hinsichtlich Arbeitsfähigkeit und körperlichen Aktivität?

- Gibt es einen Unterschied in der Arbeitsfähigkeit und körperlichen Aktivität von kaufmännischen und gewerblichen Arbeitnehmern?

\section{Methoden}

\section{Stichprobe}

Bei der Stichprobe handelt es sich um Mitarbeiter eines mittelständischen Unternehmens der Chemiebranche. Das Unternehmen gliedert sich in einen kaufmännischen Bereich, der überwiegend aus geistigen Tätigkeiten am Bildschirmarbeitsplatz besteht, und einen gewerblichen Bereich, der v. a. körperliche Tätigkeiten in der Produktherstellung umfasst. Die Rekrutierung erfolgte schriftlich über die interne Hauspost und mündlich über den Einbezug der Abteilungsleiter. Von insge- samt 578 Beschäftigten nehmen 151 Probanden $(26,12 \%)$ an der Untersuchung teil. Aufgrund von Fehlangaben in der Befragung beträgt die endgültige Personenzahl 148 (25,61\%). Es findet eine Unterscheidung in die Altersgruppen frühes berufliches AG1 (18-29 Jahre), AG2 (3049 Jahre) und AG3 (50-65 Jahre) in Anlehnung an das Statistischen Bundesamt statt [27]. Die Personendaten werden gemäß Bundesdatenschutzgesetz (insbesondere $₫ 5$ und $\S 9$ ) anonymisiert behandelt und ausgewertet. Vor der Datenerhebung musste eine Einverständniserklärung zur Nutzung der Daten seitens der Teilnehmer unterzeichnet werden.

\section{Instrumente}

Die Arbeitsfähigkeit wird mittels „Work Ability Index" (WAI) von Tuomi et al. [8] erhoben. Der Fragebogen erlaubt eine Bewertung der Arbeitsfähigkeit und definiert Ziele von Maßnahmen. Aufgrund der persönlichen Voraussetzungen der Person als auch der Gegebenheiten des Arbeitsplatzes beschreibt er, inwiefern die Person in der Lage ist, ihre Arbeit auszuführen. Für diese Untersuchung wurde die Kurzversion mit 10 Items in sieben Dimensionen genutzt. Die Bewertung der Arbeitsfähigkeit erfolgt durch Summieren der Scorepunkte jeder Dimension. Der Gesamtscore ergibt einen Wert zwischen 7 und 49 Punkten und zeigt auf, wie der Level der Arbeitsfähigkeit einzustufen ist und welche Folgerungen für die Praxis daraus resultieren (• Abb. 1, [8]).

Die körperliche Aktivität wird mithilfe des „Global Physical Activity Questionnaires" (GPAQ) erhoben, der von der WHO entwickelt wurde und aus 16 Items in vier Dimensionen besteht [2]. Er erfasst die (subjektiv empfundene) tägliche körperliche Aktivität während der Arbeit, der Freizeit sowie auf Transportwegen und ermittelt anhand dessen die tägliche körperliche Gesamtaktivität sowie die wöchentliche Gesamtaktivität, jeweils als metabolisches Äquivalent (MET). 1 MET entspricht dabei dem minütigen Energieverbrauch in Ruhe. Für diese Studie wird einzig die wöchentliche Gesamtaktivität genutzt. Das Ausmaß der körperlichen Aktivität wird in (moderat) und intensive körperliche Aktivitäten (hoch) differenziert. 
In Anlehnung an die Mindestanforderungen der WHO von 2,5 h pro Woche moderater körperlicher Aktivität ist mindestens ein moderates Aktivitätsniveau anzustreben (• Abb. 2).

\section{Durchführung}

Die Erhebung der Daten erfolgt im Rahmen des Betrieblichen Gesundheitsmanagements und wird mittels Fragebögen als Paper-und-Pencil-Test durchgeführt. Da das Unternehmen das Betriebliche Gesundheitsmanagement schon einige Jahre durchführt, haben die Beschäftigten bereits Erfahrungen mit solchen Untersuchungen. Für die Informationen und Aufklärung über die Thematik, die Fragenbögen und den Datenschutz wird daher wenig Zeit beansprucht.

Die Befragung findet durch den Gesundheitsmanager in Form kleinerer Gruppen (bis 10 Mitarbeiter) in eigens dafür vorbereiteten Räumlichkeiten statt, um gleiche Testbedingungen zu schaffen. Für die Befragung werden 20 min je Mitarbeiter eingeplant. Die eigentliche Beantwortungszeit beträgt maximal $15 \mathrm{~min}$, sodass ausreichend Zeit für eventuelle Rückfragen besteht. Mithilfe der Daten werden die Mitarbeiter hinsichtlich ihrer Tätigkeit in kaufmännische Tätigkeitsbereiche (KA) und gewerblichen Tätigkeitsbereich (GA) sowie in drei Altersgruppen (AG) eingeteilt. Die statistische Auswertung der erhobenen Daten erfolgt durch SPSS 21.0 („Statistical Package for the Social Sciences“). Die Prüfung auf signifikante Unterschiede zwischen den Tätigkeitsgruppen erfolgt mittels t-Test für unabhängige Stichproben sowie Mann-Whitney-UTest, die Prüfung zwischen den Altersgruppen mittels einfaktorieller Varianzanalyse und Poc-Host-Test (Bonferroni). Eine Normalverteilung und homogene Varianzen werden jeweils vorausgesetzt. Als Irrtumswahrscheinlichkeit wird $a<5 \%$ angenommen [17].

\section{Ergebnisse}

Das Probandengut besteht aus 116 Männern $(78,38 \%)$ und 32 Frauen $(21,62 \%)$. Der kaufmännische Tätigkeitsbereich (KA) umfasst 100 Personen (67,57\%), der gewerbliche Bereich (GA) 48 Personen
Präv Gesundheitsf 2015 • 10:199-205 DOI 10.1007/s11553-015-0496-z

๑ Die Autor(en) 2015. Dieser Artikel ist auf Springerlink.com mit Open Access verfügbar

\section{B. Biallas · P. Ashton · C. Wilke · T. Elis · I. Froböse}

Tätigkeits- und altersbezogene Analyse von Arbeitsfähigkeit und körperlicher Aktivität

\section{Zusammenfassung}

Hintergrund. Unternehmen sehen sich zunehmend vor der Herausforderung, qualifizierte Nachwuchskräfte zu finden und erfahrene, ältere Mitarbeiter bis zur Rente im Unternehmen zu halten. Arbeitsfähigkeit und körperliche Aktivität beeinflussen die Leistungsfähigkeit des Mitarbeiters. Die vorliegende Studie untersucht die Faktoren Arbeitsfähigkeit und körperliche Aktivität im Altersvergleich bei Arbeitnehmern eines mittelständischen Unternehmens.

Methode. 116 Männer (78,38\%) und 32 Frauen $(21,62 \%)$ nehmen an der Untersuchung teil. Das Durchschnittsalter der Probanden beträgt 40,85 \pm 10,07 Jahre. Die 148 Probanden gliedern sich in einen kaufmännischen Bereich $(n=100 ; 67,57 \%)$ und einen Produktionsbereich ( $n=48 ; 32,43 \%$ ). Die Arbeitsfähigkeit wird mittels „Work Ability Index", die körperliche Aktivität durch den "Global Physical Activity Questionnaire" erhoben.
Ergebnisse. Die Arbeitsfähigkeit weist einen signifikanten Unterschied $(p=0,001)$ zwischen den Berufsgruppen auf. Ein Altersunterschied besteht nicht. Die körperliche Aktivität offenbart einen signifikanten Unterschied ( $p=0,000$ ) für die Berufsgruppen, nicht jedoch für das Alter.

Schlussfolgerung. Die Förderung der körperlichen Aktivität sowie flankierende Maßnahmen zur Unterstützung der Arbeitsfähigkeit sind aus gesellschafts- und gesundheitspolitischen Gründen unumgänglich. Eine Implementierung entsprechender Maßnahmen liegt im Interesse der Arbeitnehmer, der Ar-

\section{Schlüsselwörter}

Betriebliche Gesundheitsförderung . Ältere Arbeitnehmer · Aktivitätsniveau . Demografischer Wandel · Erkrankungen, muskuloskelettale beitgeber wie der Gesellschaft

\section{Job- and age-related analysis of work ability and physical activity}

\section{Abstract}

Background. Employers are being affected by a shortage of qualified workers and, thus, are faced with the challenge of binding older employees until retirement. Physical activity (PA) and work ability are of growing importance in modern working society. This study analyses work ability and PA of employees in a medium-sized business regarding age and occupation.

Methods. The total sample consists of 148 employees (116 men, $78.38 \%$; 32 women, $21.62 \%$; mean age: $40.85 \pm 10.07$ years): 100 white-collar workers (67.57\%), and 48 bluecollar workers $(32.43 \%)$. Work ability is measured using the Work Ability Index, and PA is assessed via the Global Physical Activity Questionnaire.

Results. Work ability shows significant differences regarding occupation $(p=0.001)$ but

$(32,43 \%)$. Das Probandenalter beträgt im Mittel 40,85 $\pm 10,07$ Jahre. Die Altersgruppen umfassen 26 (AG1), 91 (AG2) und 31 Mitarbeiter (AG3). Auffällig ist, dass gewerbliche Mitarbeiter v. a. in Altersgruppe AG2 zu finden sind (• Tab. 1).

Hinsichtlich der Tätigkeit zeigen beide Gruppen eine gute Arbeitsfähigkeit. KA $(43 \pm 3,93)$ weisen jedoch eine signifikant not regarding age. Furthermore, significant differences are found for PA concerning occupation $(p=0.000)$, but again not for age. Overall, more than half of all subjects meet the current guidelines for PA.

Conclusion. Work ability is rated as good; however, special focus should lie on the promotion of PA during early and late working life. In addition, there is still not enough evidence on the prevalence of PA regarding the working society. Considering PA in working life could add to meeting current activity recommendations.

\section{Keywords}

Workplace health promotion - Activity level . Ageing workforce $\cdot$ Demographic change . Musculoskeletal diseases bessere Arbeitsfähigkeit $(p=0,001)$ als GA $(40 \pm 4,14)$ auf. Auch im Altersvergleich erreichen alle Gruppen eine gute Arbeitsfähigkeit. Gruppe AG1 erzielt einen höheren WAI-Score $(43 \pm 4,51)$ als die Gruppen AG2 $(42 \pm 3,93)$ und AG3 $(41 \pm 4,38)$. Das Ergebnis der Varianzanalyse ist jedoch nicht signifikant ( $p=0,225$, - Tab. 2). Innerhalb der Tätigkeitsbereiche wird deut- 


\section{Arbeitswelt}

\begin{tabular}{|c|c|c|c|}
\hline \multicolumn{3}{|l|}{ Variable } & Häufigkeit [n (\%)] \\
\hline \multirow[t]{3}{*}{ Geschlecht } & \multicolumn{2}{|l|}{ Männlich } & $116(78,38)$ \\
\hline & \multicolumn{2}{|l|}{ Weiblich } & $32(21,62)$ \\
\hline & \multicolumn{2}{|l|}{ Gesamt } & $148(100)$ \\
\hline \multirow[t]{11}{*}{ Alter } & \multicolumn{2}{|l|}{$x \pm s$} & $40,85 \pm 10,07$ \\
\hline & \multirow[t]{3}{*}{ 18-29 (AG1) } & Gesamt & $26(17,57)$ \\
\hline & & Kaufmännisch & $23(15,54)$ \\
\hline & & Gewerblich & $7(2,03)$ \\
\hline & \multirow[t]{3}{*}{$30-49$ (AG2) } & Gesamt & $91(61,49)$ \\
\hline & & Kaufmännisch & $53(35,81)$ \\
\hline & & Gewerblich & $38(25,68)$ \\
\hline & \multirow[t]{3}{*}{$50-65$ (AG3) } & Gesamt & $31(20,95)$ \\
\hline & & Kaufmännisch & $24(16,22)$ \\
\hline & & Gewerblich & $9(4,73)$ \\
\hline & \multicolumn{2}{|l|}{ Gesamt } & $148(100)$ \\
\hline \multirow[t]{9}{*}{ Tätigkeit } & \multirow[t]{4}{*}{ Kaufmännisch } & Gesamt & $100(67,57)$ \\
\hline & & AG1 & $23(15,54)$ \\
\hline & & AG2 & $53(35,81)$ \\
\hline & & AG3 & $24(16,22)$ \\
\hline & \multirow[t]{4}{*}{ Gewerblich } & Gesamt & $48(32,43)$ \\
\hline & & AG1 & $7(4,73)$ \\
\hline & & AG2 & $38(21,62)$ \\
\hline & & AG3 & $9(6,08)$ \\
\hline & \multicolumn{2}{|l|}{ Gesamt } & $148(100)$ \\
\hline
\end{tabular}

Tab. 2 Arbeitsfähigkeit im Tätigkeits- und Altersvergleich $(n=148)$

\begin{tabular}{|c|c|c|c|c|c|}
\hline & & & $n$ & $x \pm s$ & $p$ (2-seitig) \\
\hline \multirow[t]{17}{*}{ WAI } & \multicolumn{2}{|l|}{ KA } & 100 & $43 \pm 3,93$ & 0,001 \\
\hline & \multicolumn{2}{|l|}{ GA } & 48 & $40 \pm 4,14$ & \\
\hline & \multicolumn{2}{|l|}{ AG1 } & 26 & $43 \pm 4,51$ & 0,225 \\
\hline & \multicolumn{2}{|l|}{ AG2 } & 91 & $42 \pm 3,93$ & \\
\hline & \multicolumn{2}{|l|}{ AG3 } & 31 & $41 \pm 4,38$ & \\
\hline & \multirow[t]{3}{*}{ KA } & AG1 & 23 & $43 \pm 4,64$ & 0,563 \\
\hline & & AG2 & 51 & $43 \pm 3,40$ & \\
\hline & & AG3 & 24 & $42 \pm 4,34$ & \\
\hline & \multirow[t]{3}{*}{ GA } & AG1 & 7 & $41 \pm 3,40$ & 0,492 \\
\hline & & AG2 & 28 & $41 \pm 4,30$ & \\
\hline & & AG3 & 9 & $39 \pm 3,50$ & \\
\hline & \multirow[t]{2}{*}{ AG1 } & KA & 23 & $43 \pm 4,64$ & 0,169 \\
\hline & & GA & 10 & $41 \pm 3,40$ & \\
\hline & \multirow[t]{2}{*}{ AG2 } & KA & 51 & $43 \pm 3,40$ & 0,008 \\
\hline & & GA & 26 & $41 \pm 4,30$ & \\
\hline & \multirow[t]{2}{*}{ AG3 } & KA & 24 & $42 \pm 4,34$ & 0,037 \\
\hline & & GA & 11 & $39 \pm 3,50$ & \\
\hline
\end{tabular}

lich, dass Ältere (AG3) jeweils die niedrigste Arbeitsfähigkeit aufweisen. Signifikante Unterschiede bestehen nicht. Dagegen wird deutlich, dass der signifikante Unterschied zwischen KA und GA in den
Differenzen innerhalb der Altersgruppen AG2 und AG3 begründet liegt (• Tab. 2).

Während ein Großteil der Probanden $(42,86 \%)$ nicht die Mindestanforderungen der WHO erfüllt (niedriges Ak- tivitätsniveau), erreichen über die Hälfte $(57,14 \%)$ die Mindestanforderungen (moderates Aktivitätsniveau) oder übertreffen diese (hohes Aktivitätsniveau). Zudem wird deutlich, dass KA in geringerem Maße $(48,48 \%)$ die Mindestanforderungen erreichen als GA (75,0\%). In Bezug auf das Alter erreichen Personen aus AG2 häufiger die Mindestanforderungen $(61,11 \%)$ als AG1 (50,0\%) und AG3 (51,62\%, - Tab. 3).

In Bezug auf die Tätigkeit erreichen GA höhere Werte als KA. In der Gesamtaktivität übersteigt der Score der GA den der KA um mehr als das Dreifache. Dieser Unterschied ist signifikant $(p=0,000)$. Gleichzeitig weisen beide Gruppen eine sehr große Streuung auf (• Tab. 4). Hinsichtlich des Alters erreicht AG2 die höchste Aktivität. Zudem ist ersichtlich, dass sich Personen aus AG3 in der Gesamtaktivität von Personen aus AG1 nur geringfügig unterscheiden. Signifikante Unterschiede zwischen den Altersgruppen bestehen nicht. Analog zum Tätigkeitsvergleich sind auch bei allen drei Altersgruppen hohe Streuungen zu erkennen.

Ein Blick in die Altersgruppen innerhalb der Tätigkeitsbereiche zeigt, dass KA in allen drei Altersgruppen eine geringere Gesamtaktivität aufweisen, als GA, innerhalb der Tätigkeitsbereiche bestehen jedoch keine signifikanten Unterschiede zwischen den Altersgruppen. Es zeigt sich ferner, dass die signifikanten Unterschiede zwischen KA und GA in den Diskrepanzen der Teilnehmer im jüngeren (AG1) und mittleren Berufsalter (AG2) liegen (• Tab. 4).

\section{Diskussion}

Die KA haben eine signifikant höhere Arbeitsfähigkeit als die GA. Beide Gruppen erreichen jedoch ein gutes Niveau. Hinsichtlich des Alters lassen sich keine signifikanten Unterschiede erkennen. Betrachtet man das Aktivitätsniveau getrennt nach Tätigkeit und Altersklasse, so weisen die GA signifikant höhere Werte auf, als die KA. Dabei überragen die GA die KA um das Dreifache. 
Tab. 3 Aktivitätsniveau der Gesamtstichprobe sowie getrennt nach Tätigkeit und Altersgruppe $(n=148)$

\begin{tabular}{lllllll} 
& \multicolumn{2}{l}{ Aktivitätsniveau } & \multicolumn{3}{l}{ Hoch } \\
\cline { 2 - 7 } & Niedrig & \multicolumn{3}{l}{ Moderat } & \\
\cline { 2 - 7 } & $\boldsymbol{n}$ & $\%$ & $\boldsymbol{n}$ & $\%$ & $\boldsymbol{n}$ & $\%$ \\
\hline Gesamt & 63 & 42,86 & 36 & 24,49 & 48 & 32,65 \\
\hline KA & 51 & 51,52 & 27 & 27,27 & 21 & 21,21 \\
\hline GA & 12 & 25,00 & 9 & 18,75 & 27 & 56,25 \\
\hline AG1 & 13 & 50,00 & 3 & 11,54 & 10 & 38,46 \\
\hline AG2 & 35 & 38,89 & 25 & 27,78 & 30 & 33,33 \\
\hline AG3 & 15 & 48,38 & 8 & 25,81 & 8 & 25,81 \\
\hline n & & & & & & \\
\hline
\end{tabular}

$n$ Grundgesamtheit.

Tab. 4 Körperliche Gesamtaktivität (in MET-Minuten) im Vergleich von Tätigkeit und Alter $(n=148)$

\begin{tabular}{|c|c|c|c|c|c|}
\hline & & & $n$ & $x \pm s$ & $p$ (2-seitig) \\
\hline \multirow[t]{17}{*}{ Gesamtaktivität } & \multicolumn{2}{|l|}{ KA } & 98 & $2231,63 \pm 3654,49$ & \multirow[t]{2}{*}{0,000} \\
\hline & \multicolumn{2}{|l|}{ GA } & 47 & $7024,89 \pm 7545,97$ & \\
\hline & \multicolumn{2}{|l|}{ AG1 } & 26 & $3058,46 \pm 4673,93$ & \multirow[t]{3}{*}{0,447} \\
\hline & \multicolumn{2}{|l|}{ AG2 } & 88 & $4269,20 \pm 6246,27$ & \\
\hline & \multicolumn{2}{|l|}{ AG3 } & 31 & $3021,29 \pm 4660,62$ & \\
\hline & \multirow[t]{3}{*}{ KA } & AG1 & 26 & $2034,78 \pm 1954,21$ & \multirow[t]{3}{*}{0,875} \\
\hline & & AG2 & 51 & $2411,76 \pm 4756,63$ & \\
\hline & & AG3 & 24 & $2037,50 \pm 1826,37$ & \\
\hline & \multirow[t]{3}{*}{ GA } & AG1 & 7 & $10906,67 \pm 11,252,54$ & \multirow[t]{3}{*}{0,658} \\
\hline & & AG2 & 28 & $6829,46 \pm 7158,18$ & \\
\hline & & AG3 & 9 & $6394,29 \pm 8869,92$ & \\
\hline & \multirow[t]{2}{*}{ AG1 } & KA & 23 & $2034,781954,21$ & \multirow[t]{2}{*}{0,018} \\
\hline & & GA & 10 & $10906,6711252,54$ & \\
\hline & \multirow[t]{2}{*}{ AG2 } & $\mathrm{KA}$ & 51 & $2411,764756,63$ & \multirow[t]{2}{*}{0,002} \\
\hline & & GA & 26 & $6829,467158,18$ & \\
\hline & \multirow[t]{2}{*}{ AG3 } & $\mathrm{KA}$ & 24 & $2037,501826,37$ & \multirow[t]{2}{*}{0,170} \\
\hline & & GA & 11 & $6394,298869,92$ & \\
\hline
\end{tabular}

$n$ Größe der Grundgesamtheit, $x$ Mittelwert, $s$ Standardabweichung, $p$ Signifikanzniveau.

\section{Methoden}

Bei der vorliegenden Studie handelt es sich um eine Querschnittsuntersuchung. Die Mitarbeiter nehmen freiwillig an der Untersuchung teil, wodurch eine positive Selektion vorliegt. Es handelt sich um eine positive Selektion der Mitarbeiter, da diese freiwillig an der Untersuchung teilgenommen haben. Ferner kann nicht ausgeschlossen werden, dass v. a. aktive Personen bzw. Teilnehmer an Angeboten im Rahmen des BGM teilnehmen („healthy worker effect", [4]). An der Untersuchung nehmen 26,12\% aller Mitarbeiter teil. Vergleichbare Studien weisen in dieser Hinsicht Quoten zwischen 30 und $50 \%$ auf $[28,30]$. Aufgrund von Fehlbeantwortungen fallen 3 Probanden aus der Bewertung heraus. Der endgültige Stichprobenumfang beträgt $n=148$, was einer Auswertquote von $(98,91 \%)$ gleichkommt. Der Männeranteil an der Untersuchung $(78,38 \%)$ überwiegt deutlich jenen der Frauen (21,62\%). Die Schiefe in der Geschlechtsverteilung verhindert einen direkten Geschlechtervergleich beider Parameter. Das Alter entspricht mit 40,85 $\pm 10,07$ den Ergebnissen vergleichbarer Studien $[8,28]$. Zudem unterstreichen Studienergebnisse die Erkenntnis, dass KA häufiger an Befragungen teilnehmen, als GA [27].

Ein Diskussionspunkt bei Befragungen ist das Antworten gemäß sozialer Erwünschtheit. Die Tatsache, dass sich Mitarbeiter besser darstellen wollen ist praktisch aufgrund der Anonymisierung der
Daten und der Wahrung des Datenschutzes nichtig, theoretisch besteht der Bezug jedoch darin, dass die Leistung einer Person einen immer größeren Stellenwert in der modernen Gesellschaft einnimmt [14].

Der Einsatz des WAI wurde zwar in vielen beruflichen Settings erprobt, es besteht jedoch weiter der Bedarf nach systematischen Analysen der Bedeutung von arbeitsbezogenen und individuellen Determinanten der Arbeitsfähigkeit [29]. Die dadurch gewonnen Erkenntnisse dienen letztlich dem Design von Interventionen, um insbesondere die Arbeitsfähigkeit und die Teilhabe am beruflichen Erwerbsleben älterer Arbeitnehmer zu erheben. Bei der Erhebung der körperlichen Aktivität durch den GPAQ muss auf die erhöhte Bias-Wahrscheinlichkeit hingewiesen werden, da es sich hierbei um eine subjektive Einschätzung der Teilnehmer handelt und keine objektiven Daten zur Validierung vorhanden sind [5]. Aktuelle Studien weisen auf diese Problematik hin, erklären aber auch, dass der GPAQ akzeptabel für Populationsstudien ist und nur einen moderat bis geringen Bias aufweist [11].

\section{Ergebnisse}

Die drei Altersgruppen unterscheiden sich nicht wesentlich voneinander und erreichen jeweils eine gute Arbeitsfähigkeit ( $p=0,225$, - Tab. 2). Bisherige Studienergebnisse bekunden jedoch, dass die Wahrscheinlichkeit einer schlechteren Arbeitsfähigkeit mit steigendem Alter größer wird [27]. Auch andere Forschungen führen an, dass die Streuung der Arbeitsfähigkeit interindividuell, d. h. bei Personen gleichen Alters, altersprogressiv zunimmt, jedoch auch bei jüngeren Personen beobachtbar ist [15]. Die Standardabweichung verdeutlicht, dass die Streuung mit zunehmendem Alter vergleichsweise ab- und nicht zunimmt (• Tab. 2). Dies steht in Kontrast zu den Aussagen der Forscher, wonach die Streuung mit zunehmendem Alter wächst. Einige andere Studien werfen inzwischen ebenfalls auf, dass es keinen eindeutigen Altersunterschied hinsichtlich der Arbeitsfähigkeit gibt [8]. Wilke et al. [29] untersuchten die Auswirkungen der betrieblichen Ge- 
sundheitsförderung auf die Arbeitsfähigkeit bei jüngeren ( $<45$ Jahre) und älteren Arbeitnehmern ( $\geq 45$ Jahre). Sie postulieren ebenfalls, dass scheinbar keine Unterschiede zwischen jüngeren und älteren Mitarbeitern in ihrer Querschnittsstudie herrschen. Im Tätigkeitsvergleich besteht ein signifikanter Unterschied bezüglich der Arbeitsfähigkeit (『 Tab. 2), was bisherigen Forschungen entspricht [22, 27]. Dabei ist auffällig, dass KA v. a. im mittleren (AG2) und späten Berufsalter (AG3) eine signifikant bessere Arbeitsfähigkeit aufweisen, als GA. Dennoch weisen GA eine gute Arbeitsfähigkeit auf, und nur wenige Studien belegen bisher, dass Mitarbeiter aus körperlichen Berufsgruppen gute oder sehr gute Werte hinsichtlich der Arbeitsfähigkeit erreichen [9]. Dies muss unter Veränderungen im Anforderungsprofil von GA diskutiert werden, da aufgrund einer weitgehenden Automatisierung von Produktionsketten die körperliche Belastung deutlich reduziert ist.

Ein Großteil der Probanden (57,14\%) erfüllt die Mindestvorgaben des ACSM [14] von 2,5 h körperlicher Aktivität pro Woche. 32,65\% sind sogar körperlich hoch aktiv und übertreffen die Mindestanforderungen (• Tab. 3). Diese Erkenntnis steht bisherigen Untersuchungen zur Prävalenz körperlicher Aktivität entgegen, nach denen nur $40 \%$ der Deutschen Erwachsene im Alter von 1865 Jahren 2,5 h oder mehr pro Woche körperlich aktiv sind [25]. Im internationalen Vergleich zeigt sich ein anderes Bild. Studienergebnisse legen dar, dass der Anteil an geringer körperlicher Aktivität unterhalb von $50 \%$ für Personen aus den USA $(15,9 \%)$, Australien $(17,2 \%)$, Norwegen $(26,1 \%)$, Belgien $(43,0 \%)$ und Schweden $(23,9 \%)$ beträgt $[6,13]$.

Die vorliegende Studie entspricht in höherem Maße internationalen als nationalen Ergebnissen. Diese Ambivalenz muss in Bezug zur Nutzung subjektiver Daten und der bereits angesprochenen Biaswahrscheinlichkeit gesetzt werden, sodass eine Überschätzung der körperlichen Aktivität möglich ist, auch wenn andere Untersuchungen eine nur geringe Biaswahrscheinlichkeit aussprechen [11].

Der Vergleich der Altersgruppen verdeutlicht, dass beinahe jeder zweite junge und ältere Mitarbeiter zu wenig kör- perlich aktiv ist ( Tab. 3). Hier liegt der Schluss nahe, dass die Tätigkeit hierauf einen Einfluss hat, da wesentlich mehr KA als GA in den Gruppen AG1 und AG3 zu finden sind. Hierzu ist es jedoch in zukünftigen Studien unabdingbar, eine weitere Differenzierung in die arbeitsbezogene und freizeitliche Aktivität vorzunehmen. Gleichzeitig übertrifft nur jeder 4. ältere Mitarbeiter die Anforderungen der WHO, respektive jeder 3. für AG1 und AG2. Dies entspricht bisherigen Ergebnissen $[1,20]$.

Der Vergleich dieser Ergebnisse mit bisherigen Forschungen zeigt insgesamt, dass Ältere nicht notwendigerweise weniger körperlich aktiv sind, jedoch weniger häufig hoch aktiv sind. Insgesamt erreichen 48,48\% der KA die Mindestanforderungen, für GA sind es $75,0 \%$. GA scheinen demnach seltener niedrig aktiv bzw. öfter hoch aktiv zu sein und häufiger die Mindestanforderungen des ACSM zu erreichen als KA. Bislang zeigt sich eine Ambivalenz in der aktuellen Forschungslage zu dieser Thematik [17].

Die wöchentliche Gesamtaktivität insgesamt zeigt, dass die Probanden ein moderates körperliches Aktivitätsniveau aufweisen (『 Abb. 2, $\square$ Tab. 4). Die hohe Standardabweichung stellt gleichwohl eine hohe Disparität dar. Die Mitarbeiter des vorliegenden Unternehmens unterscheiden sich insgesamt stark in ihrer Gesamtaktivität. Dieses Ergebnis entspricht anderen Untersuchungen [10]. Im Hinblick auf das Alter lassen sich keine signifikanten Unterschiede erkennen, auch nicht innerhalb der Tätigkeitsbereiche (• Tab. 4). Dies mag an der hohen Standardabweichung in den Altersgruppen liegen, die eine hohe interindividuelle Disparität darstellt, sondern auch an den. Darüber hinaus entspricht die wöchentliche Gesamtaktivität von älteren Mitarbeitern (AG3) jener junger Mitarbeiter (AG1). Dieses Ergebnis steht in Kontrast zu bisherigen Studien, wonach ältere Erwachsene eine höhere Prävalenz für eine niedrige Aktivitätsrate aufweisen als Jüngere [6]. Hinsichtlich der Gesamtaktivität weisen GA eine dreifach höhere körperliche Aktivität als KA auf ( $p=0,000$, - Tab. 4). Mitarbeiter in diesem beruflichen Tätigkeitsfeld weisen ein allgemein großes Defizit an körperlicher Aktivität auf, was den hohen Anteil an niedrig aktiven KA erklärt (• Tab. 3). Die Mitarbeiter beider Bereiche unterscheiden sich zudem nicht nur im Hinblick auf das jeweilige Tätigkeitsfeld allgemein, sondern auch in Bezug auf das junge und mittlere Berufsalter (• Tab. 4).

Ayala et al. [3] postulieren, dass die körperliche Aktivität bei Mitarbeitern mit geistigen und körperlichen Tätigkeiten großen Schwankungen unterliegt. Eine Einordnung dieser Ergebnisse in die aktuelle Forschungslage erweist sich als schwierig, da bestehende Studien überwiegend die Subdomänen berufsbezogene, transportbezogene und/oder freizeitbezogene körperliche Aktivität im Hinblick auf die Berufsgruppen untersuchen, nicht aber die Gesamtaktivität [17, 20, 25]. Offen bleibt, ob die allgemein geforderte Aktivitätserhöhung auch im betrieblichen Setting gesundheitsfördernd wirkt oder ob nicht eine erhöhte Aktivität zu einer Mehrbelastung, und damit zu einem höheren gesundheitlichen Risiko insbesondere für körperlich Tätige, führt.

\section{Fazit für die Praxis}

Die vorliegende Untersuchung zeigt keinen Einfluss des Alters auf die Arbeitsfähigkeit und körperliche Aktivität bei Erwerbstätigen auf. Dementgegen gibt es deutliche Unterschiede in diesen Faktoren hinsichtlich der Tätigkeit. Kaufmännische Angestellte sind weisen zwar eine höhere Arbeitsfähigkeit auf, sind jedoch deutlich inaktiver als gewerbliche Angestellte. Dies ist v. a. auf die Unterschiede im mittleren und späten Berufsalter (Arbeitsfähigkeit) bzw. jungen und mittleren Berufsalter (körperliche Aktivität) zurückzuführen. Dies sollte u. a. durch die Validierung mit objektiven Daten und die Differenzierung der körperlichen Aktivität in berufsbezogene, freizeitliche und alltägliche Aktivität überprüft und auf weitere UnternehmensgröBen und Branchen in Deutschland ausgeweitet werden. Denn es mangelt bislang an der Evidenz zur Prävalenz von körperlicher Aktivität und Arbeitsfähigkeit von erwerbstätigen Erwachsenen in Deutschland. 


\section{Korrespondenzadresse

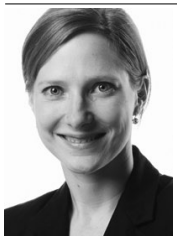 \\ B. Biallas \\ Institut für \\ Bewegungstherapie und bewegungsorientierte \\ Prävention und Rehabilitation \\ Deutsche Sporthochschule \\ Köln, Am Sportpark \\ Müngersdorf 6, 50933 Köln \\ biallas@dshs-koeln.de}

\section{Einhaltung ethischer Richtlinien}

Interessenkonflikt. Bianca Biallas, Philip Ashton, Christiane Wilke, Tobias Elis und Ingo Froböse geben an, dass kein Interessenskonflikt besteht.

Dieser Beitrag beinhaltet keine Studien an Menschen oder Tieren.

Open Access Dieser Artikel unterliegt den Bedingungen der Creative Commons Attribution License. Dadurch sind die Nutzung, Verteilung und Reproduktion erlaubt, sofern der/die Originalautor/ en und die Quelle angegeben sind.

\section{Literatur}

1. Allender S, Foster C, Boxer A (2008) Occupational and nonoccupational physical activity and the social determinants of physical activity: results from the health survey for England. J Phys Act Health 5(1):104-116

2. Armstrong T, Bull F (2006) Development of the world health organization Global Physical Activity Questionnaire (GPAO). J Public Health 14(2):66-70

3. Ayala GX, Gammelgard A, Sallis JF, Elder JP (2011) The association of physical activity and work-related characteristics among Latino adults. J Phys Act Health 8(1):79-84

4. Baillargeon J (2001) Characteristics of the healthy worker effect. Occup Med 16(2):359-366

5. Barberio A, McLaren L (2011) Occupational physical activity and body mass index (BMI) among Canadian adults: does physical activity at work help to explain the socio-economic patterning of body weight? Can J Public Health 102(3):169-173

6. Bauman A, Ma G, Cuevas F, Omar Z, Waqanivalu T, Phongsavan $P$ (2010) Cross-national comparisons of socioeconomic differences in the prevalence of leisure-time and occupational physical activity, and active commuting in six Asia-Pacific countries. J Epidemiol Community Health 65(1):35-43

7. Bechmann S, Dahms V, Tschersich N, Frei M, Leber U, Schwengler B (2012) Fachkrafte und unbesetzte Stellen in einer alternden Gesellschaft: Problemlagen und betriebliche Reaktionen. Institut für Arbeitsmarkt-und Berufsforschung (IAB), Nürnberg

8. van den Berg TIJ, Elders LAM, Zwart BCH de, Burdorf A (2008) The effects of work-related and individual factors on the Work Ability Index: a systematic review. Occup Environ Med 66(4):211-220

9. Bridger RS, Bennett Al (2011) Age and BMI interact to determine work ability in seafarers. Occup Med (Lond) 61(3):157-162
10. Charreire $H$, Kesse-Guyot $E$, Bertrais $S$, Simon $C$, Chaix B, Weber C (2011) Associations between dietary patterns, physical activity (leisure-time and occupational) and television viewing in middleaged French adults. Br J Nutr 105(6):902-910

11. Cleland CL, Hunter RF, Kee F, Cupples, ME, Sallis JF, Tully MA (2014) Validity of the Global Physical Activity Questionnaire (GPAQ) in assessing levels and change in moderate-vigorous physical activity and sedentary behaviour. BMC Public Health 14(1):1255

12. Froböse I, Wilke C, Ashton P (2012) Gesundheitsmanagement. In: Allmer H, Becker R (Hrsg) Betriebliches Gesundheitsmanagement und Prävention arbeitsbedingter Gesundheitsverfahren, Bd 36 - Demographischer Wandel Grundlagen, Ergebnisse, Maßnahmen. BKK Bundesverband, Essen

13. Hagströmer M, Oja P, Sjöström M (2007) Physical activity and inactivity in an adult population assessed by accelerometry. Med Sci Sports Exerc 39(9):1502-1508

14. Haskell W, Lee I, Pate R, Powell K, Blair S, Franklin B (2007) Physical activity and public health. Med Sci Sports Exerc 39(8):1423-1434

15. Hasselhorn H, Freude G (2007) Der Work-ability-Index: Ein Leitfaden Schriftenreihe der Bundesanstalt für Arbeitsschutz und Arbeitsmedizin/Sonderschrift: S87. Wirtschaftsverl NW, Verl für Neue Wiss, Bremerhaven

16. Hoffmann U, Orthmann P (2009) Schnellkurs Statistik mit Hinweisen zur SPSS-Benutzung (6, überarb und erw Aufl). Sport Buch Strauß, Köln

17. Holtermann A, Jorgensen MB, Gram B, Christensen JR, Faber A, Overgaard K (2010) Worksite interventions for preventing physical deterioration among employees in job groups with high physical work demands: background, design and conceptual model of FINALE. BMC Public Health 10:120

18. Ilmarinen J, Tuomi K (2004) Past present and future of work ability. In Ilmarinen J, Lehtinen S, Past present and Future of Work Ability - People and Work Research Report 65, Finnish Institute of Occupational Health, Helsinki, S 1-25

19. Joiko K, Schmauder M, Wolff G (2010) Psychische Belastung und Beanspruchung im Berufsleben Erkennen - Gestalten, 5. Aufl. Bundesanstalt für Arbeitsschutz und Arbeitsmedizin, Dortmund

20. Krug S, Jordan S, Mensink G, Müters S, Finger J, Lampert T (2013) Körperliche Aktivität. Bundesgesundheitsblatt Gesundheitsforschung Gesundheitsschutz 56(5-6):765-771

21. Kruger J, Yore MM, Ainsworth BE, Macera CA (2006) Is participation in occupational physical activity associated with lifestyle physical activity levels? J Occup Environ Med 48(11):1143-1148

22. Lahelma $E$ (2005) Occupational class inequalities across key domains of health: results from the Helsinki Health Study. Eur J Public Health 15(5):504510

23. Lange A (2012) Bewältigung von Herausforderungen des demografischen Wandels: Voraussetzungen und Ergebnisse von Demografiemanagement Erkenntnisse aus einer Reihe von Abschlussarbeiten (No 12) Working Paper Series, Chair of Organization, Human Resource General Management, Brandenburg University of Technology

24. Powell K, Paluch A, Blair S (2011) Physical activity for health: what kind? How much? How intense? On top of what? Public Health 32(1):349

25. Robert Koch-Institut Daten und Fakten (2012) Ergebnisse der Studie "Gesundheit in Deutschland aktuell 2010" Beiträge zur Gesundheitsberichterstattung des Bundes Berlin
26. Statistisches Bundesamt Bevölkerung Deutschlands bis 2060 (2009) 12 koordinierte Bevölkerungsvorausberechnung Wiesbaden

27. Tuomi K, Huuhtanen P, Nykyri E, Ilmarinen J (2001) Promotion of work ability, the quality of work and retirement. Occup Med 51(5):318-324

28. Wilke C, Krämer K, Biallas B, Froböse I (2012a) Lebensqualität und körperliche Aktivität im betrieblichen Kontext. Präv Gesundheitsf 7(1):56-61

29. Wilke C, Weber C, Biallas B, Froböse I (2012b) Betriebliche Gesundheitsförderung: Auswirkung auf die Belegschaft eines Mittelstandsbetriebs. Z Betriebliche Prävention und Unfallversicherung 9:138-145

30. Wilke C, Nagel R, Biallas B, Froböse I (2013) Gesundheitskompetenz von Mitarbeitern eines mittelständischen Unternehmens in Abhängigkeit von sportlicher Aktivität. BG Beweg Gesundh 29(02):51-54 